\title{
Differential Microbial Assemblages Associated with Shikonin-producing Borage Species in Two Distinct Soil Types
}

\section{Aliya Fazal}

Nanjing University

Minkai Yang

Nanjing University

Zhongling Wen

Nanjing University

Farman Ali

Nanjing University

Ran Ren

Nanjing University

Chenyu Hao

Nanjing University

Xingyu Chen

Nanjing University

Jiangyan Fu

Nanjing University

Xuan Wang

Nanjing University

Wencai Jie

Nanjing University

Tongming Yin

Nanjing Forestry University

Guihua Lu

Huaiyin Normal University

Jinliang Qi

Nanjing University

Yonghua Yang ( $\nabla$ yangyh@nju.edu.cn )

Nanjing University 
Keywords: HPLC, Microbial recruitment, PacBio sequencing, Shikonin

Posted Date: February 24th, 2021

DOI: https://doi.org/10.21203/rs.3.rs-233342/v1

License: (c) (1) This work is licensed under a Creative Commons Attribution 4.0 International License. Read Full License 


\section{Abstract}

Shikonin and its derivatives are the main components of traditional Chinese medicine, Zicao. The pharmacological potential of shikonin and its derivatives have been extensively studied. Yet, less is known about the microbial assemblages associated with shikonin producing Borage plants. We studied microbial profiles of two Borage species, Echium plantagineum (EP) and Lithospermum erythrorhizon (LE), to identify the dynamics of microbial colonization pattern within three rhizo-compatments and two distinct soil types. Results of $\alpha$ and $\beta$-diversity via PacBio sequencing revealed significantly higher microbial richness and diversity in the natural soil along with a decreasing microbial gradient across rhizosphere to endosphere. Our results displayed genotype and soil type-dependent fine-tuning of microbial profiles. The host plant was found to exert effects on the physical and chemical properties of soil, resulting in reproducibly different micro-biota. Analysis of differentially abundant microbial OTUs displayed, Planctomycetes and Bacteroidetes to be specifically enriched in EP and LE rhizosphere while endosphere was mostly prevailed by Cyanobacteria. Network analysis to unfold co-existing microbial species displayed different types of positive and negative interactions within different communities. The baseline data provided here will help to identify microbes associated with different rhizo-compartments of potential host plants. In the future, this might be helpful for manipulating the keystone microbes for ecosystem functioning.

\section{Introduction}

Changes in exudate composition trigger shifts in structure and function of microbial communities in soil. Such effect may feedback on plants (plant-soil feedback, PSF), altering plant metabolic pathways that retroactively modify exudate composition ${ }^{1}$. This suggests that PSF derive mechanisms through which they either regulate microbial growth or benefit plants by enhancing assimilation and nutrient uptake from soil ${ }^{2}$. Understanding PSF is critical as these mechanisms do not exist in isolation. Consequently, its vital to understand PSF and its effects on plant populations and communities. In addition to PSF, insights into microbe-microbe interactions are equally crucial. As microbes can co-occur or exclude each other thus making them another principle drivers of population structure and dynamics ${ }^{3}$.

Plants are known to harbor a distinct array of microbes within spatial locations, i.e. rhizosphere, rhizoplane, endosphere ${ }^{4}$. Studies demonstrate that the microbial diversity and abundance in all the three rhizo-compartments is shaped by plant genotype, plant age, and environmental variables such as physical and chemical properties of soil ${ }^{5}$. The characterization of complex associations between plants, root exudates, microbial communities, and environmental factors are extensively studied in model plants 6 . However, the mechanisms regarding non-model (invasive/non-invasive plants) are poorly addressed.

Echium plantagineum (EP) is a drought tolerant, noxious, and an economically important weed. In Australia, it is considered an invasive species after it was introduced in the early 1800's. EP has a greater genetic diversity, smaller genome size $(1 \mathrm{C}=0.34 \mathrm{pg})$, and exhibit an annual life cycle ${ }^{7}$. Its inter-genera Lithospermum erythrorhizon (LE), is known for its dried roots as a perpetual ingredient in Chinese 
medicine for possessing a multitude of antiviral and biological activities ${ }^{8}$. Both the species belong to the family Boraginaceae whose members are known to produce two interesting groups of secondary metabolites: pyrrolizidine alkaloids synthesized in above-ground plant tissues, and naphthoquinones (NQs) produced in roots and root hairs ${ }^{9}$.

NQs are known for their broad range of biological effects. Shikonins (SK), and its derivatives are the chief NQs that are biosynthesized via combined shikimate/mevalonate pathway ${ }^{10}$. SK is found to have varying degrees of biological activity against a wide range of soil-borne microorganisms ${ }^{11}$. It has been regarded as "biochemical novel weapons" as its uniform placement in the root hairs suggests its role as a chemical barrier to microbial invasion ${ }^{12}$.

Microbial communities associated with Borage plants have not been explored. Therefore the rationale behind this research is to utilize PacBio sequencing platform in order to; $\nabla$ unveil the associated microbial profiles of two Borage species; $\varangle$ analyze how community profiles differ among two inter-genera despite of the same growth/soil conditions; and $\nabla$ identify the rare bacterial taxa in three rhizo-compartments and the nature of feedbacks that exist between co-occuring microbial species. It was hypothesized that plant rhizo-compartments include/exclude microbes from their surrounding soil resulting in reproducibly distinct microbial community. Such selection varies with soil physical and chemical properties. Also among residing microbial communities, different microbes exert different selection pressures on neighbouring microbial communities depending upon resource availability and space. Providing such a detailed study will help to assess the nature and dynamics of microbes associated with rhizosphere and roots of potentially important Borage species. This will also help to better understand the mechanisms that affect the dynamics of associated microbial communities

\section{Results}

\section{Metabolic profiling of EP and LE root exudates and root periderm samples}

High performance liquid chromatography (HPLC) analysis of root exudates and root periderm extracts reported the presence of five bioactive NQs including shikonin (SK), acetylshikonin (AS);

isobutyrylshikonin (IBS); $\beta$, $\beta$-dimethylacrylshikonin (DMAS); and isovalerylshikonin (IVS) in both the soil types (Fig. 1a-d; Fig. S5). This suggests significant accumulation of SK and its derivatives in the rhizosphere via root exudation. Regardless of variation among samples, SK, AS, DMAS, and IVS were consistently present among all the samples.

\section{PacBio sequence reads statistics and taxonomic profiling}

After quality filtering, removal of chimera, chloroplast and mitochondrial sequences, approximately 165,570 high quality sequences (Tags) were obtained. Tags were clustered after normalization into 
14,429 microbial operational taxonomic units (OTUs) at a $97 \%$ sequence similarity cutoff level (Table S2). All OTUs with species annotation are summarized in Table S3. Taxonomic profiling for taxonomic affiliations revealed Proteobacteria, Bacteroidetes, Planctomycetes, Cyanobacteria, Acidobacteria, and Actinobacteria to be the dominant phyla among all the samples. These 6 phyla accounted for 71.84$96.61 \%$ of the total microbial OTUs (Fig. S6). The Proteobacterial microbes mainly belonged to Classes Alphaproteobacteria, Betaproteobacteria, and Gammaproteobacteria that accounted for $13.94-40.54 \%$ of the total microbes (Table S4).

\section{Host plant genetics are the drivers for distinct microbiome}

To identify the effects of host plant genetics on microbial acquisition, microbial community composition of bulk soil was compared with root and rhizosphere-associated soils of EP and LE. a-diversity estimates revealed a significantly higher observed species richness (Sobs), and shannon diversity for bulk soil (Fig. $3 a, b ;$ Table S5). This indicates that bulk soil serves as a reservoir for microbial acquisition in other rhizocompartments. At different taxonomic levels, microbes associated with Proteobacteria, Planctomycetes, Bacteroidetes and Cyanobacteria were all present in relatively higher abundance in EP and LE rhizocompartments compared to bulk soil in two different soil types (Fig. 2a; Table S6). Wilcox test displayed differential microbial acquisition when bulk soil and rhizosphere communities were compared at order level. For example, Flavobacteriales, Sphingomonadales, and Verrucomicrobiales had a relatively higher abundance in EP rhizosphere, while Caulobacterales, and Sphingomonadales were significantly higher in LE rhizosphere soil (Fig. S7, $P<0.05$ ).

The above results also corroborate with the Venn diagram where only 67 , and 122 microbial OTUs for root (endosphere + rhizoplane) and rhizosphere compartments of both EP, and LE were shared with bulk soil (Figs. 2b, c). However, 8,188 unique OTUs (4,357 OTUs for EP and 3,831 for LE) were specifically found in the root and rhizosphere zones (Table S7). This indicates that both the borages scrutinize microbes at the root-soil interface resulting in a distinct microbial community.

\section{Microbial enrichment/de-richment vary by soil type}

To investigate the influence of soil source on the root (endosphere + rhizoplane) and rhizosphere associated-microbiome, EP and LE species were grown in pots filled with natural campus (NC) and peat potting artificial (PP) soil under axenic conditions. Focusing on soil types and controlling for rhizocompartments, measures of a-diversity (Sobs, Shannon index) revealed significant differences in microbial communities. Results showed that NC soil was significantly higher for microbial richness and diversity compared to PP soil (Fig. 3a, b; Table S8). This indicates that roots of both the borage species when grown in different soil types have different effects on bacterial community dynamics.

Venn diagram was also in accordance with above results where microbial OTUs enrichment was pronounced for NC soil as only $31 \%$ OTUs in EP, and $42.7 \%$ OTUs in LE were found in PP soil samples 
(Fig. S8). Additionally, Headmap was constructed using unweighted Unifrac distance (UUF) metric to consider the taxonomic relatedness of rare taxa. The obtained results displayed considerably different taxonomic relatedness in two distinct soils (Fig. S9). These results display soil-dependent variations in microbial communities where soil physical and chemical properties contributed to such variations.

Besides differences in a-diversity, there were differences in microbial taxonomic profiles as well. For example, Proteobacteria/ microbes were significantly enriched in NC soil while PP soil had higher abundance of Planctomycetes and Bacteroidetes (Fig. 2a). Among these phyla, Burkholderiales, Chitinophagales and Planctomycetales were the orders responsible for causing significant variation. For example, microbes belonging to Burkholderiales were significantly enriched in NC soil while PP soil was considerably abundant with Chitinophagales and Planctomycetales (Fig. 4a, b; Table S9). Notably, the associated microbes responded differently to different plant species despite of the same soil type. For example, Chitinophaga costaii was successful at colonizing LE rhizo-compartments while Planctomycetal OTUs associated with Schlesneria paludicola were abundantly present in EP compartments displaying inter-genus effects (Fig. 4c; Table S10).

\section{Variation in microbial diversity within different plant niches}

Plants posses different microbial communities within diffferent rhizo-compartments (niches) (Fig. 4c). Within these niches, rhizosphere microbes tightly adhere to the roots while those of rhizoplane resides the root surface. Endosphere/endophytic compartment is composed of microbiomes that inhabit the root center. Focusing on rhizo-compartments, measures of a-diversity revealed a significantly higher richness and diversity for rhizosphere followed by rhizoplane, while endosphere had the lowest diversiy index ( $P<$ 0.05) (Fig. 3a, b; Table S11). To further analyze variations among different communities ( $\beta$-diversity), 2dimentional Principal Co-ordinate Analysis (PCoA) using Weighted UniFrac metrics (WUF) was conducted. WUF metric indicated that different rhizo-compartments (PC1) represented largest source of variation (41.00\%), followed by soil type (PC2) that explained $26.24 \%$ variation, while plant species (PC3) were responsible for causing $10.05 \%$ of total variation (Fig. 5a, b). By comparing the distances, it was observed that endosphere samples had a distinct community clustering, while rhizosphere, rhizoplane and bulk soil samples were clustered together displaying overlapping communities. Also clear separation of PP and NC soil samples was observed (Fig. 4a).

At different phylogenetic levels, Kruskul-Wallis test using microbial relative abundance displayed Proteobacteria, Bacteroidetes, Planctomycetes, and Acidobacteria to be significantly enriched in rhizosphere and rhizoplane while Cyanobacteria was abundant in endosphere (Table S12). Among Acidobacteria, Acidobacteriales occupied rhizosphere, rhizoplane (Fig. 6a, $P<0.05$ ) while enriched Cyanobacteria/microbes residing endosphere mainly belonged Nostocales which were significantly excluded from rhizosphere, rhizoplane (Fig. $6 \mathrm{~b}, P<0.05$ ). These results indicate a selective criteria at each rhizo-compartment where plants select some microbes from the surrounding while exclude others. 


\section{Analysis of differentially abundant microbes among EP and LE rhizo-compartments}

To identify the microbes that are responsible for causing community separation among different rhizocompartments of EP and LE species, top 20 differentially abundant microbial OTUs were analyzed. It was observed that microbial OTU6 (Rubinisphaera), 0TU26 (Sphingobium), and OTU17 (Phycisphaera), specifically dominated the EP rhizosphere soil while OTU1 (Chitinophaga), OTU5 (Pseudomonas), OTU46 (Terrimonas), and OTU41 (Polaromonas) predominantly occupied LE rhizosphere. Among them, Rubinisphaera brasiliensis (OTU6) of Planctomycetes was the dominant microbe in EP rhizosphere, while Chitinophaga costaii of Bacteroidetes (OTU1) was predominantly enriched in LE rhizosphere. The inner core endosphere was mostly prevailed by microbial OTU4, and OTU53, that represented microbes belonging to phylum Cyanobacteria (Fig. S10). In case of EP, the endosphere also contained Actinobacterial OTU36 that represented members of the order Actinoplanes. These results specify that the core microbial community occupying EP and LE-rhizocompartments comprises of Planctomycetes, Bacteroidetes and Cyanobacteria (Fig. 7).

\section{Co-occurring species associated with two Borage's rhizo- compartments}

While analyzing differentially enriched microbes, there were also some microbes that co-occurred among the rhizocompartments of both EP and LE. For example, among top 20 microbial OTUs, there were 12 OTUs that were equally successful at colonizing all the compartments of both EP and LE causing noteworthy overlaps in community structure and composition. The predominance of these microbes was mainly due to the enrichment of genera Variovorax (OTU22, OTU84), Pirellula (OTU11), Methylibium (OTU58), Tellurimicrobium (OTU35), Cupriavidus (OTU23, OTU30), Methylobacillus (OTU28, OTU29), Loriellopsis (OTU53), Sphingobium (OTU24), and OTU4 of phylum Cyanobacteria (genus not available) respectively (Fig. S10).

To get deeper insights, species network analysis was performed that identified positively and negatively co-occurring microbial species. Our results displayed that microbes belonging to classes Chitinophagia, and Gamma-proteobacteria were negatively co-related with neighbouring microbial species while Planctomycetia, Alpha and Beta-proteobacteria all co-existed positively (Fig. S11; Table S13). Moving on, a total of top 10 highly negatively and positively co-related microbes were considered. Obtained results revealed that Chitinophaga costaii, Chitinophaga terrae and Dyella japonica were the species that were negatively co-related with majority of other bacterial species, while Pirellula staleyi, Novosphingobium naphthalenivorans and Ramlibacter nginsenosidimutans outcompeted the negative ones and positively co-occurred with the neighboring species (Fig. 8; $P<0.05$ ).

It is worthy to mention that the microbes having a negative co-relation with their neighbors were found to be positively associated with each other. For example, Heatmap with a correlation coefficient greater than 
0.2 showed a strong positive correlation of Dyella japonica with Chitinophaga costaii and Chitinophaga terrae (Fig. S12). These results suggest that positive and negative feedbacks occur among co-occuring microbial communities. Within that feedback, some micro-organisms imply minimal competition for resources while others offer maximum resistance resulting in a definite microbial assemblage.

\section{Discussion}

Plant-soil feedback (PSF) is a complex mechanism that involves interaction of biotic and abiotic drivers. PSF can range from positive to negative that ultimately affect plant performance and soil community dynamics ${ }^{1}$. Among the biotic factors, host plant age and genetics background can considerably affect composition of complex microbial communities ${ }^{13}$. In our study, microbial community dynamics of two shikonin-producing inter-genera Borages were analyzed and the results displayed that the two plant species were found to harbor a distinct microbial population recruited from the surrounding bulk soil (Fig.2). This indicates a selection criteria at the soil-root interface where plant species scrutinize microbes resulting in a distinct community. Such selective recruitment suggests a promising role of plant root exudates in specific microbial selection. Similar results were observed by Mosqueira, et al. ${ }^{14}$ where bacterial communities were recruited from the surrounding bulk soil. Hirsch and Mauchline ${ }^{15}$ also reported the uptake of bacterial and fungal communities from the surrounding soil to the root surface.

In addition to biotic factors, physicochemical factors like $\mathrm{pH}$, temperature, trophic resources, and soil texture are responsible for microbial community composition turnover ${ }^{16}$. While studying the microbial communities of tobacco plant, Wang, et al. ${ }^{17}$ reported shifts in soil microbial community composition in response to soil physico-chemical properties. In a similar study, soil type and plant genotype played a key role for causing variation in rhizobacterial community of cotton ${ }^{18}$. In our study, a significant difference in the $\alpha-, \beta$-diversity, and taxonomic profile of bacterial communities was observed among natural campus (NC) and peat potting artificial (PP) soil. This indicates that soil microbial background is a major factor for variable microbial composition in two soil types. In addition, our results displayed that despite of the same soil type, the associated microbes responded differently to different plant species (Fig. 4). For example, in PP soil, E. plantagineum rhizosphere was dominated by uncultivated members of the phylum Planctomycetes while sequences affiliated with Bacteroidetes were mostly abundant in L. erythrorhizon rhizosphere. This signifies host genotype, and soil type-dependent fine tuning of microbial profiles depending upon soil biological and chemical properties.

Plants are found to harbor distinguished bacterial communities depending upon plant tissue and rhizocompartment ${ }^{19}$. Three distinct root-associated compartments have been identified which are specific habitats for microbial colonization ${ }^{20}$. Within these habitats, microbes are assembled largely from the surrounding environment. Coleman-Derr, et al. ${ }^{21}$ demonstrated that different plant compartments of Agave species are primarily occupied by variable proportions of prokaryotic communities. Another study by Chen, et al. ${ }^{22}$ reported that the plant compartments had a significant role in affecting the bacterial and fungal compositions of Broussonetia papyrifera seedlings where an increasing diversity gradient was 
observed from the interior root to the exterior rhizosphere. In our study, we were able to characterize the composition of each of the three rhizocompartments. It was observed that each of the rhizocompartment was found to contain a distinct bacterial community where, rhizosphere had the highest bacterial diversity than endosphere (Fig 3 ). This suggests that plants exert exclusionary effects on specific microbes. Such effects initiate at the rhizosphere, then rhizoplane allows the microbes to enter the root interior i.e. endosphere.

Microbes in a particular environment compete with other microbes for resource availability. Such competitions also allow them to make complex communities that play roles in maintaining healthy associations with their hosts ${ }^{23}$. To reveal such intricate microbial associations, network-based approaches are employed that allow us to gain novel insights of microbial interactions within communities ${ }^{24}$. Shaw, et al. ${ }^{25}$ by utilizing species network analysis demonstrated that, the addition of $S$. acidaminiphila changed the overall composition of native microbial communities thus concluding that subtle perturbation changes the dynamics of core microbiome. In our study, microbial species cooccurrence patterns were analyzed in order to understand the synergistic relationships among microbes residing different rhizo-compartments. It was observed that Chitinophagia were found to co-exist with Gamma-Proteobacteria, while Planctomycetia co-occurred with Alpha and Beta-Proteobacteria (Fig. 8). The co-occurrence of Proteobacteria with Bacteroidetes and Planctomycetes indicates their dominant in a wide range of soils. Ling, et al. ${ }^{26}$ reported the presence of Proteobacteria, Cyanobacteria, Bacteroidetes, and Actinobacteria in the rhizosphere soils of watermelon. Proteobacteria, Actinobacteria and, Bacteroidetes were also the dominant phyla in the rhizosphere soil of transgenic maize carrying mcry genes ${ }^{27}$. These reports validate that Proteobacteria/microbes offer minimal range of competition to other bacterial species for resources.

There are several studies regarding Cyanobacteria to be equally beneficial to host plants ${ }^{28}$. For example, some members of Nostocales and others cyanobacterial strains are known to secrete active products that trigger the release of plant secondary products by influencing endogenous hormone level. This ultimately helps host plant to modify its surrounding environment ${ }^{29,30}$. Also microbes belonging to Actinobacteria are important plant probiotics as they possess antimicrobial activities against pathogenic fungi or bacteria ${ }^{31}$. In our study, root interior was found to selectively recruit bacteria affiliated with Loriellopsis (Cyanobacteria) and Actinoplanes (Actinobacteria) while rhizosphere was mostly prevailed by Proteobacteria, Planctomycetes and Bacteroidetes. Such selective recruitment suggests that rhizocompartments are the dominant factor in shaping microbial assemblages. Previous studies also reported the role of rhizocompartments in affecting bacterial community structure of maize ${ }^{32}$. Lee, et al. ${ }^{19}$ also reported varied microbial composition in response to plant compartment with a distinct community in endosphere.

\section{Conclusion}


Our results provide a detailed characterization of the microbiome of shikonin-producing borages via PacBio sequencing. Both the Borage species were found to harbor a genotype and soil type-dependent distinct microbiome. Baseline data provided here proved that each soil has its microbial pool from where a plant draws the most favorable microbial OTUs to organize its microbes. In addition, all the three rhizocompartments (rhizosphere, rhizoplane, endosphere) were found to contain a distinct array of microbes where rhizosphere had the highest species diversity. The distribution of microbial taxa in specific plant compartments will help to further improve our understandings of plant-microbiome interactions in realworld. In future, such information would be useful to manipulate the roles of identified microbes by microbial engineering to increase plant productivity of potential host plants.

\section{Materials And Methods \\ Plant propagation}

Mature E. plantagineum (EP) and L. erythrorhizon (LE) seeds were collected from the field in Inner Mongolia Autonomous Region, China. Permissions were obtained before collection. Seeds were washed with sterile distilled water, followed by $75 \%$ ethanol for $5 \mathrm{~min}$, and then were germinated on petri plates at $25 / 18^{\circ} \mathrm{C}$ day/night temperatures with a 12 -h photoperiod. Afterwards, $7 \mathrm{~d}$-old healthy seedlings were aseptically transplanted into pots filled with two chemically distinct soils.

\section{Soil type, collection and properties}

Two kinds of soils were used in our experiment. $\otimes$ Peat potting artificial soil (PP) (Klasmann, Germany), $\nabla$ Natural campus soil (NC). Soil was taken at the sampling depth of $10 \mathrm{~cm}$. PP soil was black peat moss type soil with $\mathrm{pH} 6.34$; SOM $1.20 \%$; $\mathrm{N} 0.97 \% ; \mathrm{P} 0.7 \%$; $\mathrm{K} 0.6 \%$, while $\mathrm{NC}$ soil had a loamy texture with $\mathrm{pH}$ 7.85; SOM 1.305\%; N 0.233\%; P 0.58\%; K 1.305\%. Five seedlings per pot (three replicates per treatment) were used. Soil in pots devoid of plants served as bulk soil control to differentiate plant effects from general edaphic factors. All the plant specimens were propagated for almost 15-18 weeks (Fig. S1) under controlled greenhouse conditions at 25/18 C day/night temperatures ${ }^{33}$.

\section{Chemical extraction of shikonin and its derivatives from root exudates and root samples}

For chemical extraction, greenhouse grown specimens were collected in compliance with relevant institutional, national, and international guidelines and legislation. For trapping root exudates, a customized static culture-based system adopted from Phillips, et al. ${ }^{34}$ was used (Fig. S2). Root samples were prepared by carefully excavating, and placing roots in moist paper towel for $24 \mathrm{~h}$ at $4^{\circ} \mathrm{C}$ prior extraction. For avoiding plant-to-plant variation, composite samples were prepared from 3-4 individual plant roots for each sample. $\sim 0.2 \mathrm{~g}$ fresh root periderm peels were extracted in $100 \% \mathrm{HPLC}$ grade ethanol 
on an orbital shaker at $120 \mathrm{rpm}$ in dark, at room temperature for $14 \mathrm{~h}$. Following extraction, samples were filtered using a $0.45 \mu \mathrm{m}$ syringe filter, and $10 \mu \mathrm{L}$ of each extract was transferred to HPLC vials for High performance liquid chromatography (HPLC) analysis ${ }^{33}$ via HPLC Agilent 1200 Series (Agilent Technologies, USA). Samples were loaded on $\mathrm{C}_{18}$ Thermo Hypersil Gold column $(4.6 \times 250 \mathrm{~mm}, 5 \mu \mathrm{m}$, Welch Materials, Inc, China) with a flow rate of $1.0 \mathrm{ml} \mathrm{min}^{-1}$ and run time of $30 \mathrm{~min}$. A gradient of mobile phase $A\left(\mathrm{H}_{2} \mathrm{O}+0.1 \%\right.$ formic acid) and mobile phase $B(95 \%$ acetonitrile $+0.1 \%$ formic acid) with a ratio of 30:70. Peaks were identified using analytical standards of shikonin ( $\left.S K_{M w} 288.0997 ; R_{t}=5.3 \mathrm{~min}\right)$, acetylshikonin ( $A S_{M W}$ 330.1103; RT 7.5 min); isobutylshikonin ( $I B S_{M W} 358.4 ; R_{t}=11.8 \mathrm{~min}$ ); $\beta$, $\beta$ dimethylacrylshikonin (DMAS ${ }_{M W} 370.1416$; RT $13.55 \mathrm{~min}$ ); and isovalerylshikonin (IVS $\mathrm{MW}_{372.4 ;} \mathrm{R}_{\mathrm{t}}=14.67$ min) purchased from Nanjing Puyi Biotechnology Co., Ltd. Nanjing, China.

\section{Microbial DNA extraction}

The rhizosphere (RS), rhizoplane (RP) and endophytic compartment (EC)/endosphere samples were prepared as described by Edwards, et al. ${ }^{4}$ (Fig. S3-S4). DNA from RS and RP soil samples was extracted using Power Soil@ DNA Isolation kit (MoBio Laboratories, US). For EC samples, roots were first grounded to a fine powder using liquid nitrogen prior DNA extraction. DNA quality was assessed and quantified on $1 \%$ agarose gel using Qubit 2.0 Fluorometer (Invitrogen, Carlsbad, USA).

\section{Library construction and Third generation sequencing platform}

Pacific Biosciences (PacBio) Single Molecule, Real-Time (SMRT) DNA sequencing (Pacific Biosciences, Shenzhen, China) (http://www.pacb.com) of full 16S rRNA gene was employed for determining bacterial community structure and composition of bulk, root, and soil samples. 16S rRNA gene was amplified by KAPA HiFi hot start DNA polymerase from $50 \mathrm{ng}$ of genomic DNA using the barcoded bacterial-specific primer 27F (5'-AGRGTTYGATYMTGGCTCAG) and 1492R (5'-RGYTACCTTGTTACGACTT) with 30 cycles of $95^{\circ} \mathrm{C}$ denaturation $30 \mathrm{sec}, 57^{\circ} \mathrm{C}$ annealing $30 \mathrm{sec}$, and $72{ }^{\circ} \mathrm{C}$ extension $60 \mathrm{sec}$. The resulting PacBio libraries were sequenced on a PacBio Sequel platform ${ }^{35}$. A total of 544,206 circular consensus sequence (CCS) reads were produced which were then filtered out using barcode information that produced a total of 165,570 high quality amplicon sequences (Tags) (Table S1). The obtained effective Tags were analyzed using the JGI iTag analysis pipeline (iTagger v.1.1) ${ }^{36}$ and clustered into OTUs (operational taxonomic units) using a $97 \%$ cutoff. Filtering, chimera detection and clustering were performed using a set of MOTHUR tools ${ }^{37}$. Chimeras were removed by filtering reads $\leq 1340$ and $\geq 1640$ bp using reformat.sh in BBMap ${ }^{35}$. After the chimeras were removed, chloroplast, and mitochondrial sequences were discarded using the RDP-classifier ${ }^{38}$. Classification of clusters was achieved by alignment to the Greengenes database (13.8.99) ${ }^{39}$. After sequencing data was rarefied and normalized, alpha and beta diversity analyses were performed for characterizing bacterial richness and diversity. Raw sequence data 
file is available in NCBI Sequence Read Archive in FASTQ format with the study accession numbers under Bio-project PRJNA664270.

\section{Statistical analysis}

All analyses were performed using Mothur software (version: 1.39 .5$)^{37}$ with R package for corresponding Graphs/histograms for $\mathrm{a}, \beta$-diversity estimates, and determination of abundances at different taxonomic hierarchies. Differences in bacterial community composition were based on Bray-Curtis, weighted UniFrac (WUF), and unweighted Unifrac (UUF) distances (phyloseq package). Results for $\beta$-diversity analysis were visualized by Principle Coordinate Analysis (PCoA) using WUF distance metrix. Significant differences in relative abundances or in a-diversity indices were determined by employing ANOVA Tukey's test, KruskalWallis, and Wilcoxon Rank-Sum Test. Headmaps were constructed using UUF, and WUF metrices. UUF considers the taxonomic relatedness of rare taxa, while WUF metrix takes the abundance of taxa into consideration. Cytoscape network analysis based on the abundance of bacterial species was performed using R software (v3.4.1) for co-occurring species analysis.

\section{Declarations}

\section{Conflict of interest}

The authors declare no potential conflict of interest. The funding sponsors had no role in the design of the study, collection, analyses, interpretation of the data, writing of the manuscript, and in the decision to publish the results.

\section{Acknowledgments}

This work was supported by the grants from the National Natural Science Foundation of China (U1903201, 31771413, 31670298), and the Program for Changjiang Scholars and Innovative Research Team in University from the Ministry of Education of China (IRT_14R27).

\section{References}

1. Bennett, J. A. et al. Plant-soil feedbacks and mycorrhizal type influence temperate forest population dynamics. Science. 355, 181-181 https://doi.org/10.1126/science.aai8212 (2017).

2. Canarini, A., Kaiser, C., Merchant, A., Richter, A. \& Wanek, W. Root Exudation of Primary Metabolites: Mechanisms and Their Roles in Plant Responses to Environmental Stimuli (vol 10, 157, 2019). Front Plant Sci 10, doi:ARTN 42010.3389/fpls 2019.00420 (2019).

3. Falony, G. et al. Population-level analysis of gut microbiome variation. Science. 352, 560-564 https://doi.org/10.1126/science.aad3503 (2016). 
4. Edwards, J. et al. Structure, variation, and assembly of the root-associated microbiomes of rice. $P$ Natl Acad Sci USA. 112, E911-E920 https://doi.org/10.1073/pnas.1414592112 (2015).

5. Marques, J. M. et al. Plant age and genotype affect the bacterial community composition in the tuber rhizosphere of field-grown sweet potato plants. FEMS Microbiol. Ecol. 88, 424-435 https://doi.org/10.1111/1574-6941.12313 (2014).

6. Donn, S., Kirkegaard, J. A., Perera, G., Richardson, A. E. \& Watt, M. Evolution of bacterial communities in the wheat crop rhizosphere. Environ Microbiol. 17, 610-621 https://doi.org/10.1111/14622920.12452 (2015).

7. Zhu, X. C. et al. Ecology and genetics affect relative invasion success of two Echium species in southern Australia (March, 42792, 2017). Sci Rep-Uk 7, doi:ARTN 4478710.1038/srep44787(2017).

8. Lin, H. Y. et al. Design, synthesis and biological evaluation of shikonin thio-glycoside derivatives: new anti-tubulin agents. Rsc Adv. 4, 49796-49805 https://doi.org/10.1039/c4ra08810g (2014).

9. Callaway, R. M. \& Ridenour, W. M. Novel weapons: invasive success and the evolution of increased competitive ability. Front Ecol Environ. 2, 436-443 doi:Doi 10.1890/15409295(2004)002[0436:Nwisat]2.0.Co;2 (2004).

10. Papageorgiou, V. P., Assimopoulou, A. N., Couladouros, E. A., Hepworth, D. \& Nicolaou, K. C. The chemistry and biology of alkannin, shikonin, and related naphthazarin natural products. Angew Chem Int Edit. 38, 270-301 (1999).

11. Li, M. Y., Xu, Z. T., Zhu, C. L. \& Wang, J. Effect of Different Derivatives of Shikonin from Lithospermum erythrorhizon Against the Pathogenic Dental Bacteria. Curr Pharm Anal. 8, 255-260 https://doi.org/10.2174/157341212801619324 (2012).

12. Brigham, L. A., Michaels, P. J. \& Flores, H. E. Cell-specific production and antimicrobial activity of naphthoquinones in roots of Lithospermum erythrorhizon. Plant Physiol. 119, 417-428 (1999). DOI 10.1104/pp.119.2.417

13. Zogg, G. P., Travis, S. E. \& Brazeau, D. A. Strong associations between plant genotypes and bacterial communities in a natural salt marsh. Ecol Evol. 8, 4721-4730 https://doi.org/10.1002/ece3.4105 (2018).

14. Mosqueira, M. J. et al. Consistent bacterial selection by date palm root system across heterogeneous desert oasis agroecosystems. Sci Rep-Uk 9, doi:ARTN 403310.1038/s41598-019-40551-4(2019).

15. Hirsch, P. R. \& Mauchline, T. H. Who's who in the plant root microbiome? Nat Biotechnol. 30, 961-962 https://doi.org/10.1038/nbt.2387 (2012).

16. Neilson, J. W. et al. Significant Impacts of Increasing Aridity on the Arid Soil Microbiome.Msystems2, doi:ARTN e00195-1610.1128/mSystems.00195 - 16 (2017).

17. Wang, R. et al. Microbial community composition is related to soil biological and chemical properties and bacterial wilt outbreak. Sci Rep-Uk 7, doi:ARTN 34310.1038/s41598-017-00472-6(2017).

18. Qiao, Q. H. et al. The Variation in the Rhizosphere Microbiome of Cotton with Soil Type, Genotype and Developmental Stage. Sci Rep-Uk 7, doi:ARTN 394010.1038/s41598-017-04213-7(2017). 
19. Lee, S. A. et al. A preliminary examination of bacterial, archaeal, and fungal communities inhabiting different rhizocompartments of tomato plants under real-world environments. Sci Rep-Uk 9, doi:ARTN 930010.1038/s41598-019-45660-8(2019).

20. Jeewani, P. H. et al. Shifts in the bacterial community along with root-associated compartments of maize as affected by goethite. Biol Fert Soils. https://doi.org/10.1007/s00374-020-01458-9 (2020).

21. Coleman-Derr, D. et al. Plant compartment and biogeography affect microbiome composition in cultivated and native Agave species. New Phytol. 209, 798-811 https://doi.org/10.1111/nph.13697 (2016).

22. Chen, P. L. et al. The effect of plant compartments on the Broussonetia papyrifera-associated fungal and bacterial communities. Appl Microbiol Biot. 104, 3627-3641 https://doi.org/10.1007/s00253020-10466-6 (2020).

23. Yang, P. S., Yu, S. J., Cheng, L. \& Ning, K. Meta-network: optimized species-species network analysis for microbial communities. Bmc Genomics 20, doi:ARTN 18710.1186/s12864-019-5471-1(2019).

24. Hurwitz, B. L., Westveld, A. H., Brum, J. R. \& Sullivan, M. B. Modeling ecological drivers in marine viral communities using comparative metagenomics and network analyses. P Natl Acad Sci USA. 111, 10714-10719 https://doi.org/10.1073/pnas.1319778111 (2014).

25. Shaw, G. T. W. et al. A network-based approach to deciphering a dynamic microbiome's response to a subtle perturbation. Sci Rep-Uk 10, doi:ARTN 1953010.1038/s41598-020-73920-5(2020).

26. Ling, N. et al. The response of root-associated bacterial community to the grafting of watermelon. Plant Soil. 391, 253-264 https://doi.org/10.1007/s11104-015-2399-3 (2015).

27. Fazal, A. et al. Assembly and shifts of the bacterial rhizobiome of field grown transgenic maize line carrying mcry $1 \mathrm{Ab}$ and mcry2Ab genes at different developmental stages. Plant Growth Regul. 91, 113-126 https://doi.org/10.1007/s10725-020-00591-7 (2020).

28. Suarez-Moo, P. D., Vovides, A. P., Griffith, M. P., Barona-Gomez, F. \& Cibrian-Jaramillo, A. Unlocking a high bacterial diversity in the coralloid root microbiome from the cycad genus Dioon. Plos One 14, doi:ARTN e021127110.1371/journal.pone.0211271(2019).

29. Abinandan, S., Subashchandrabose, S. R., Venkateswarlu, K. \& Megharaj, M. Soil microalgae and cyanobacteria: the biotechnological potential in the maintenance of soil fertility and health. Crit Rev Biotechnol. 39, 981-998 https://doi.org/10.1080/07388551.2019.1654972 (2019).

30. Sergeeva, E., Liaimer, A. \& Bergman, B. Evidence for production of the phytohormone indole-3-acetic acid by cyanobacteria. Planta. 215, 229-238 https://doi.org/10.1007/s00425-002-0749-x (2002).

31. Singh, R. \& Dubey, A. K. Diversity and Applications of Endophytic Actinobacteria of Plants in Special and Other Ecological Niches. Front Microbiol 9, doi:ARTN 176710.3389/fmicb 2018.01767 (2018).

32. Silva, U. C. et al. Long-Term Rock Phosphate Fertilization Impacts the Microbial Communities of Maize Rhizosphere. Front Microbio/ 8, doi:ARTN 126610.3389/fmicb. 2017.01266 (2017).

33. Zhu, X. C. et al. Identification and localization of bioactive naphthoquinones in the roots and rhizosphere of Paterson's curse (Echium plantagineum), a noxious invader. J Exp Bot. 67, 37773788 https://doi.org/10.1093/jxb/erw182 (2016). 
34. Phillips, R. P., Erlitz, Y., Bier, R. \& Bernhardt, E. S. New approach for capturing soluble root exudates in forest soils. Funct Ecol. 22, 990-999 https://doi.org/10.1111/j.1365-2435.2008.01495.x (2008).

35. Singer, E. et al. High-resolution phylogenetic microbial community profiling. Isme J. 10, 2020-2032 https://doi.org/10.1038/ismej.2015.249 (2016).

36. Tremblay, J. et al. Primer and platform effects on 16S rRNA tag sequencing. Front Microbio/6, doi:ARTN 77110.3389/fmicb. 2015.00771 (2015).

37. Schloss, P. D. et al. Introducing mothur: Open-Source, Platform-Independent, Community-Supported Software for Describing and Comparing Microbial Communities. Appl Environ Microb. 75, 7537$7541 \mathrm{https}: / /$ doi.org/10.1128/Aem.01541-09 (2009).

38. Wang, Q., Garrity, G. M., Tiedje, J. M. \& Cole, J. R. Naive Bayesian classifier for rapid assignment of rRNA sequences into the new bacterial taxonomy. Appl Environ Microb. 73, 5261-5267 https://doi.org/10.1128/Aem.00062-07 (2007).

39. DeSantis, T. Z. et al. Greengenes, a chimera-checked 16S rRNA gene database and workbench compatible with ARB. Appl Environ Microb. 72, 5069-5072 https://doi.org/10.1128/Aem.03006-05 (2006).

\section{Figures}




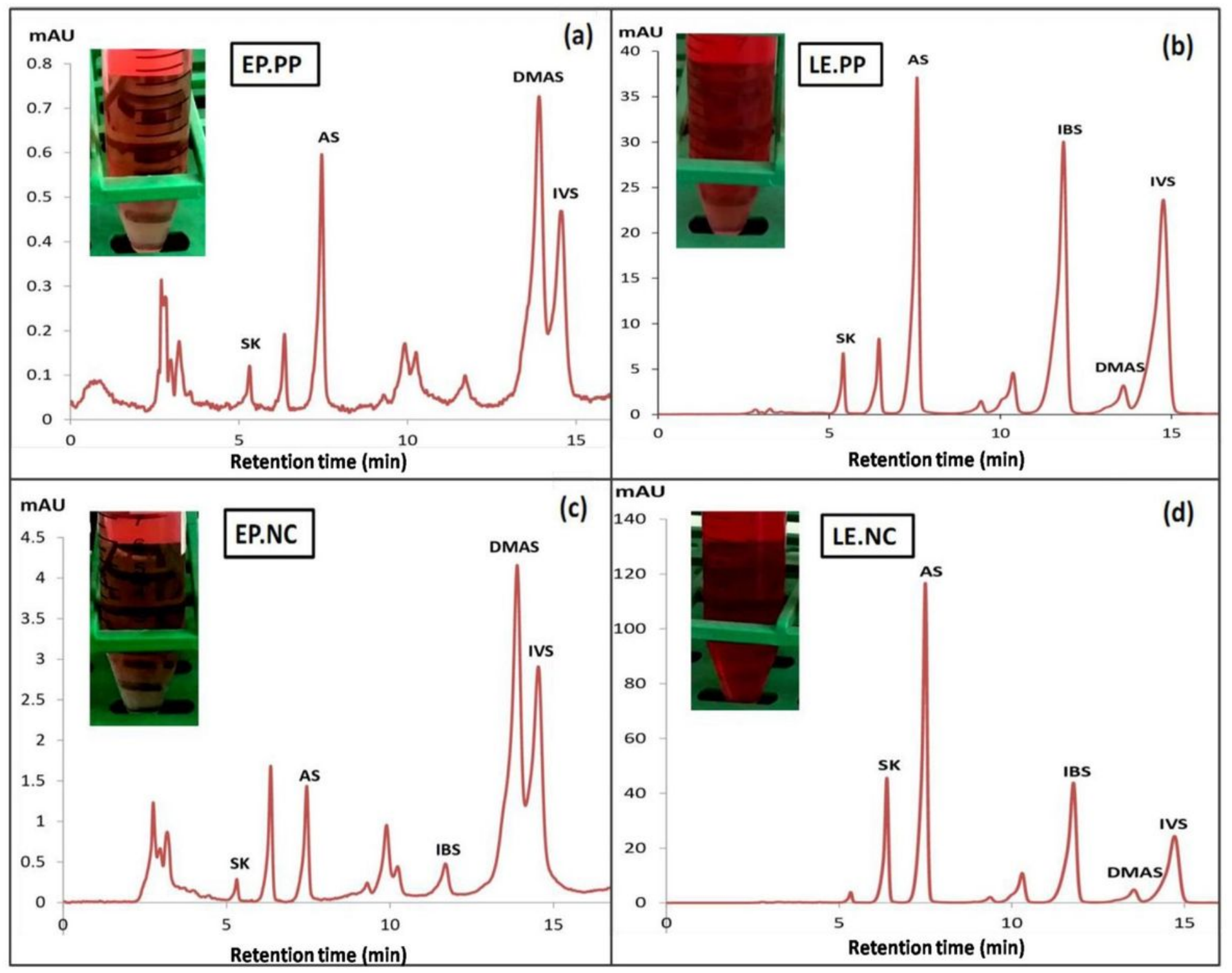

Figure 1

Images and chromatograms representing qualitative and quantitative variation of SK and its derivatives production in root periderm peel extracts. Chromatograms of root extracts obtained for E. plantagenium (EP) and L.erythrorhizon (LE) specimens via HPLC Agilent 1200 series, grown in Peat potting artificial soil (a) EP.PP (b) LE.PP; and Natural campus soil (c) EP.NC (d) LE.NC. Resulting peaks correspond to shikonin (SK), acetylshikonin (AS); isobutylshikonin (IBS); $\beta, \beta$-dimethylacrylshikonin (DMAS); and isovalerylshikonin (IVS) 
(a)

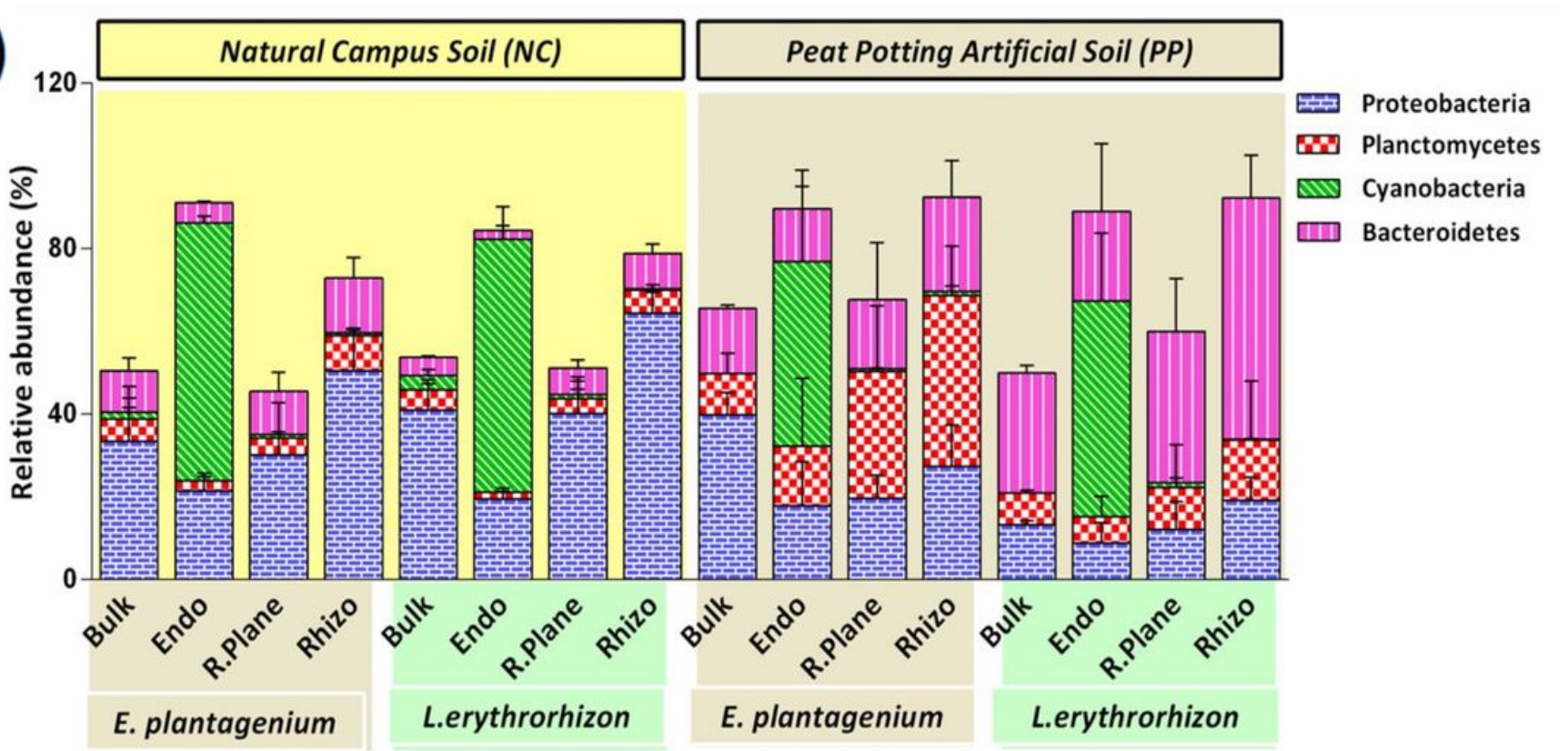

(b)

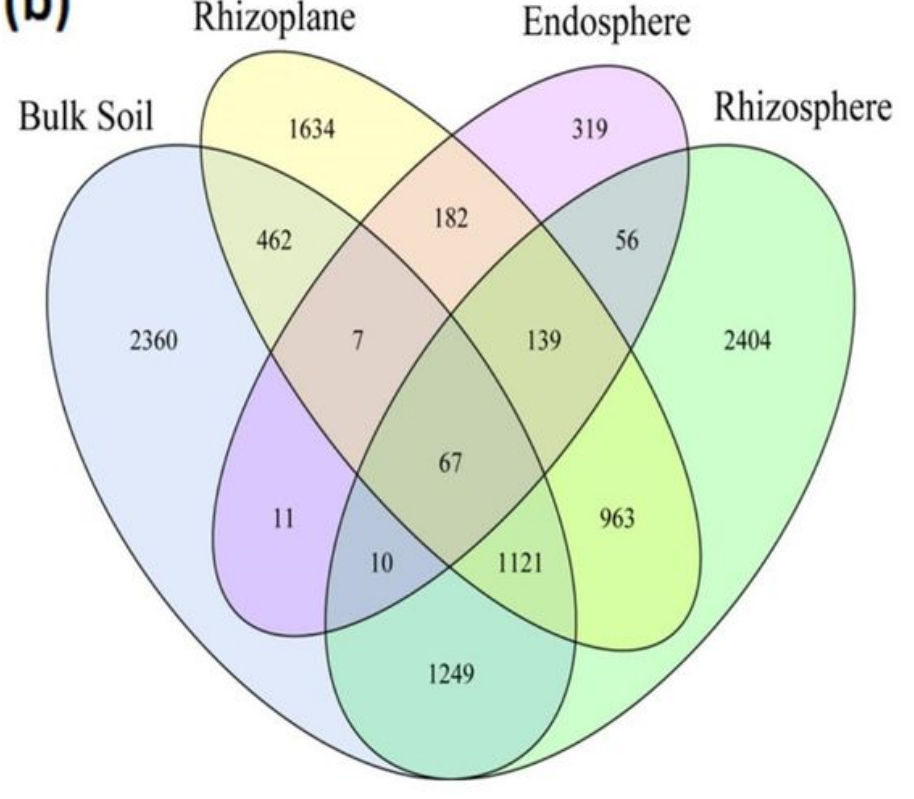

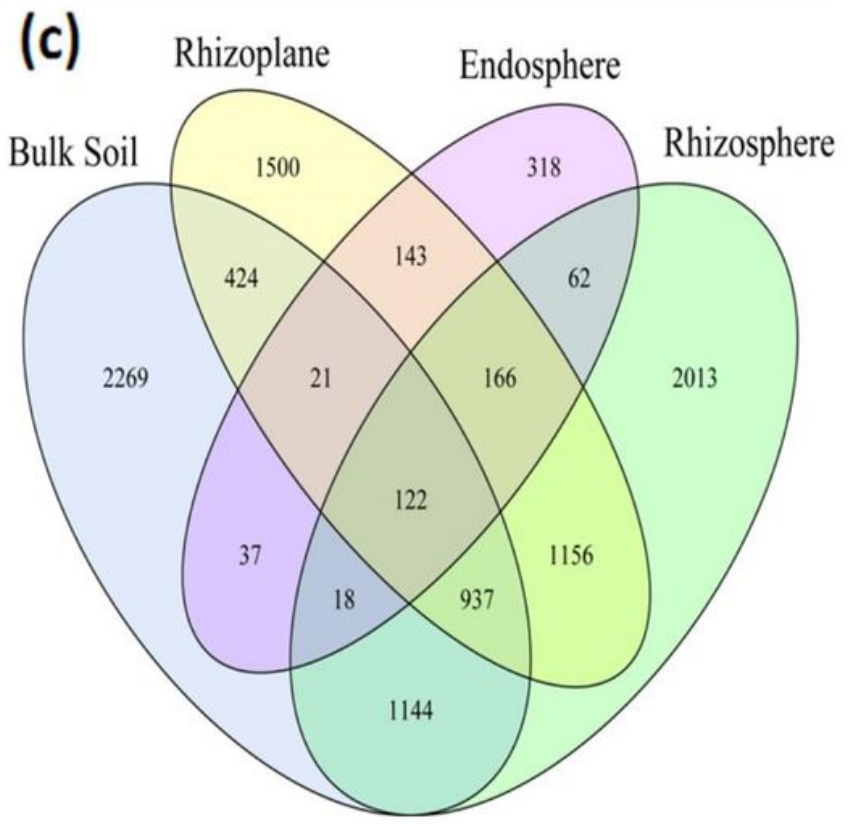

Figure 2

(a) Stacked bar graph showing relative abundances of differentially abundant microbes in each rhizocompartment (Endosphere, Rhizosphere, Rhizoplane) compared to bulk soil at Phylum level. Venn diagram showing number of specifically and commonly abundant OTUs in different compartments and bulk soil of (b) E. plantagenium, and (c) L.erythrorhizon 

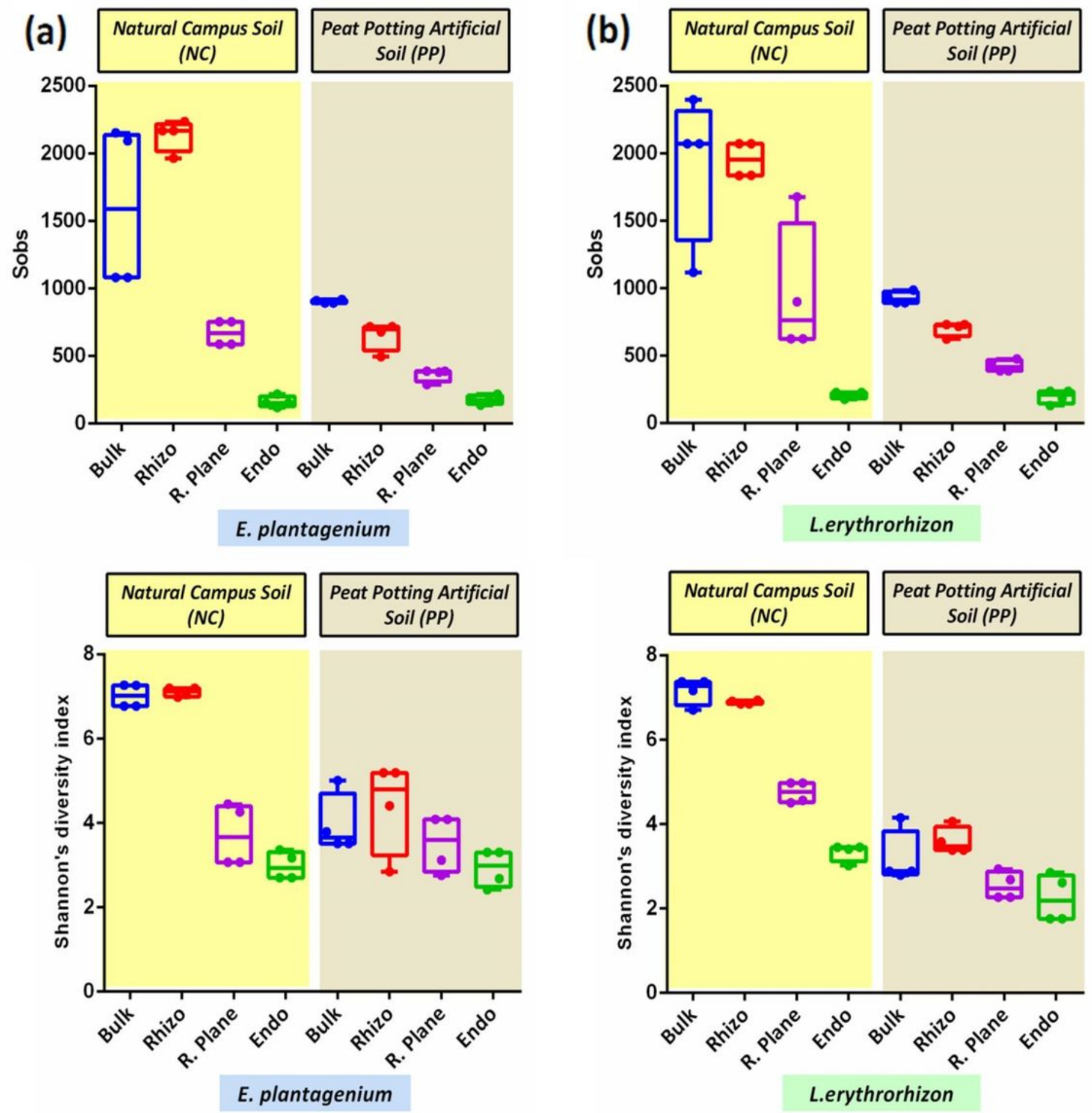

Figure 3

Boxplot of alpha diversity indices for bacterial communities among two different soil types and three rhizo-compartments. From top to bottom are Sobs, Shannon diversity index for (a) E.plantagineum; (b) L. erythrorhizon 


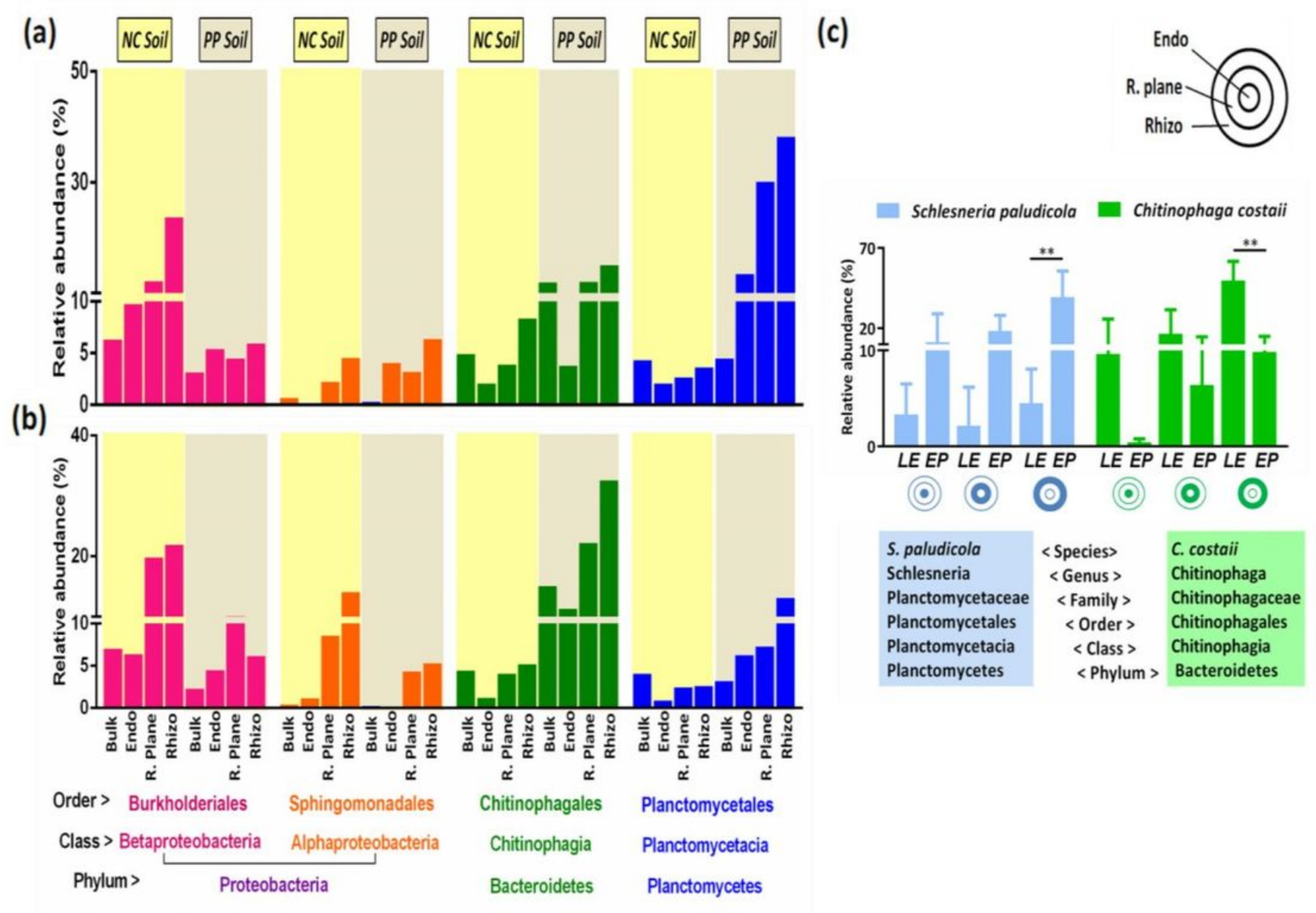

\section{Figure 4}

Differentially enriched/deriched OTUs at Order level between (a) E.plantagineum (EP), (b) L. erythrorhizon (LE) under the influence of natural campus (NC) and peat potting artificial (PP) soil. (c) Differentially expressed species in rhizosphere, rhizoplane, endosphere compartments of both EP and LE under the influence of PP soil only. ** represents significant difference $(P<0.005)$ among microbes colonizing EP and LE rhizosphere 

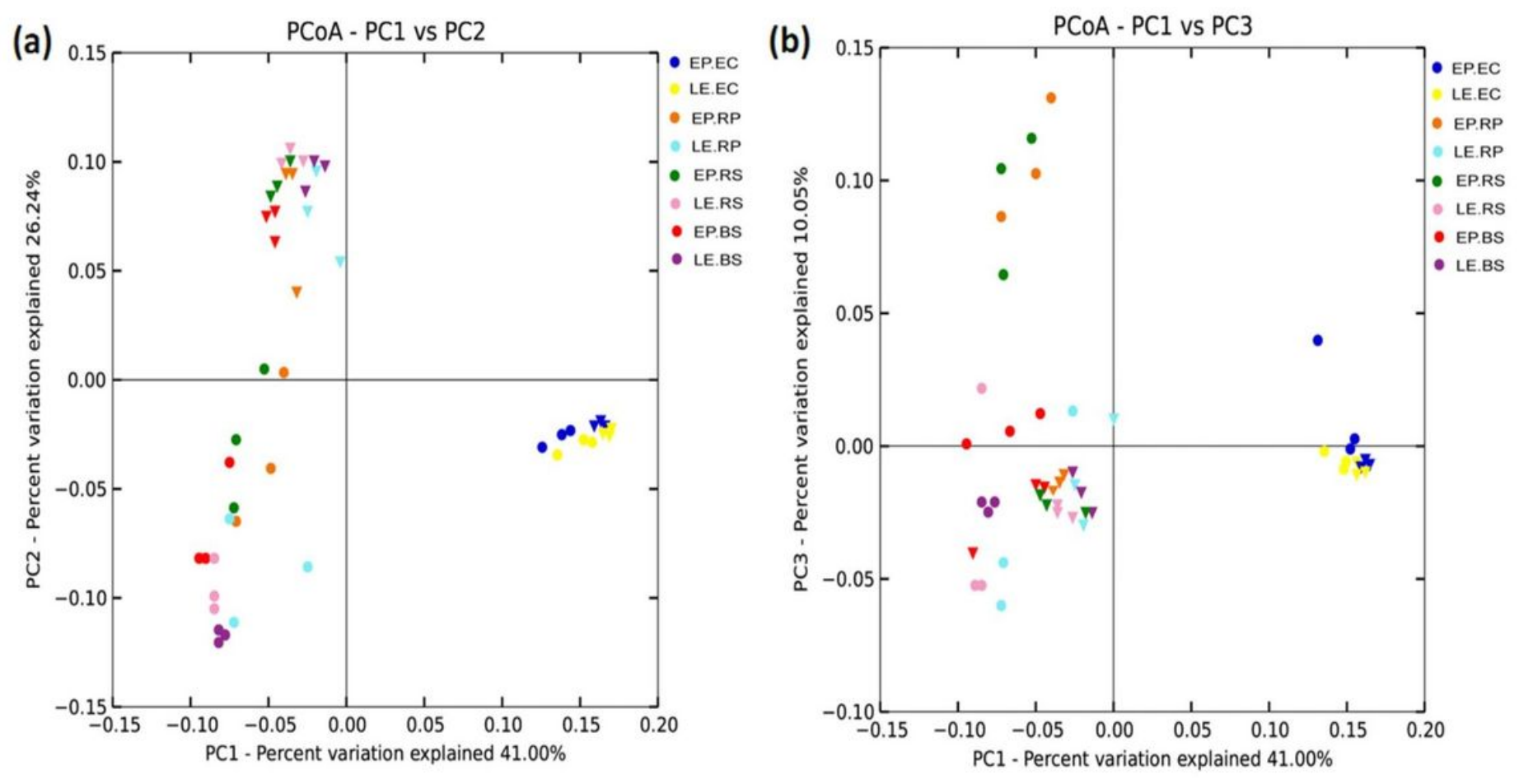

Figure 5

$\beta$-diversity analysis using WUF-PCoA that represents microbial separation depending upon three rhizocompartments i.e. endophytic compartment/endosphere (EC), rhizoplane (RP), rhizosphere (RS); soil type i.e. Nanjing campus (NC), and peat potting (PP); and plant species i.e. E. plantagineum (EP), and L. erythrorhizon (LE). Object shape represents soil type; circle (PP), triangle (NC). Color represents different rhizo-compartments 

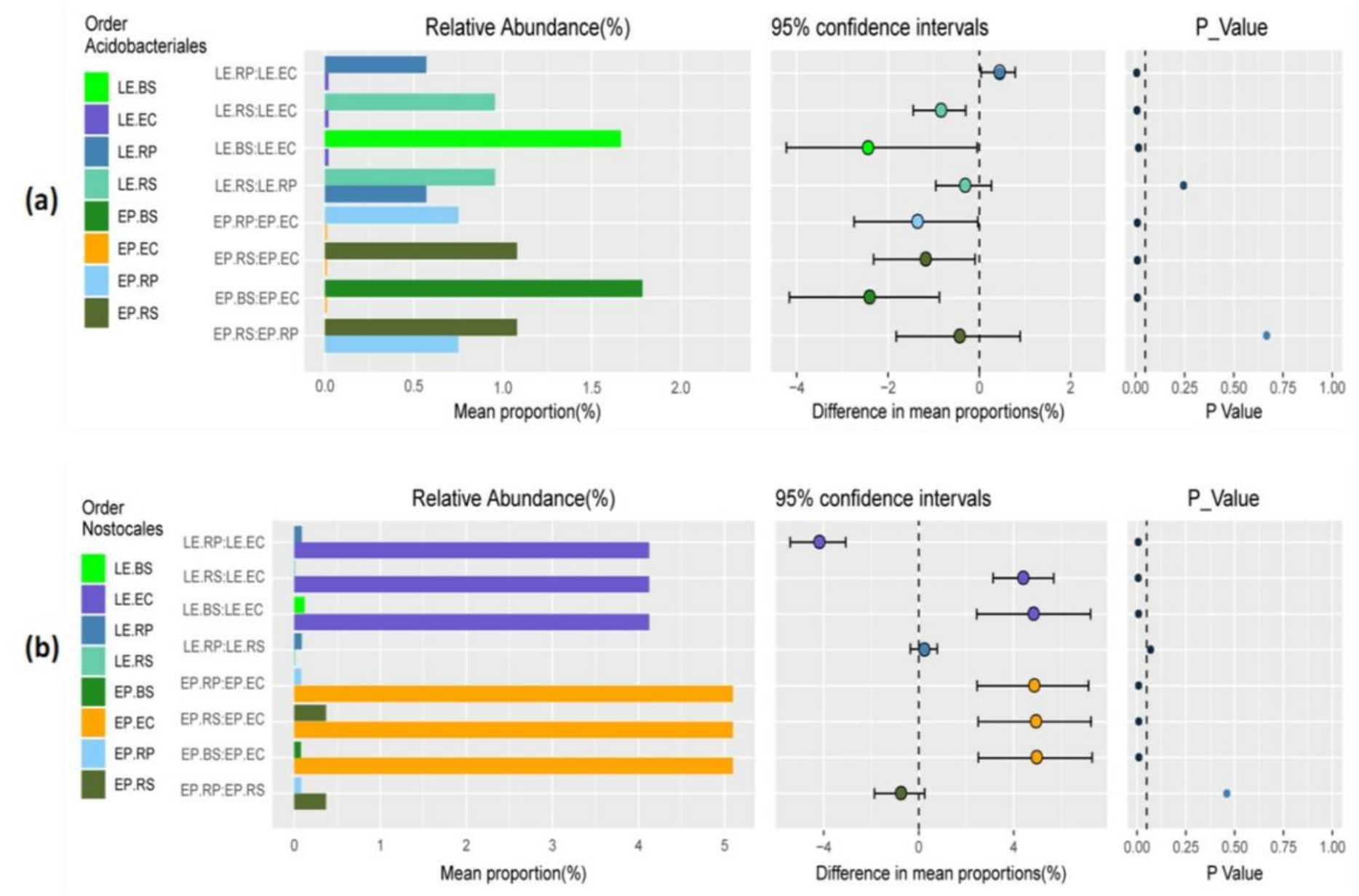

\section{Figure 6}

Kruskul-Wallis test using relative abundance of microbes at order (a) Acidobacteriales; (b) Nostocales residing endophytic compartment $(\mathrm{EC})$, rhizoplane (RP), rhizosphere (RS), and bulk soil compartments of E. plantagineum (EP), and L. erythrorhizon (LE) plant species 


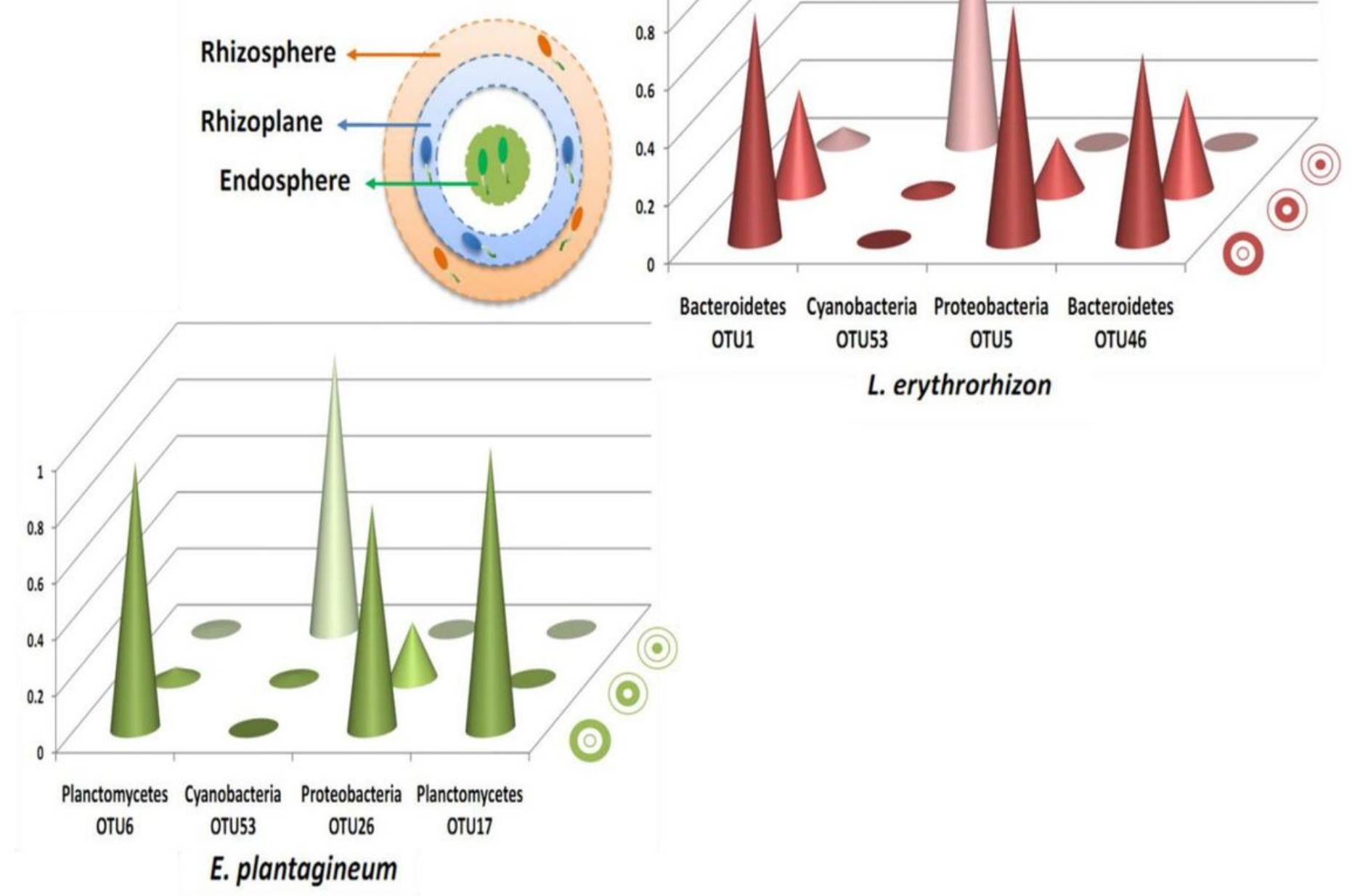

Figure 7

A proposed model representing core microbiome associated with E. plantagineum and L. erythrorhizon species within three rhizo-compartments 


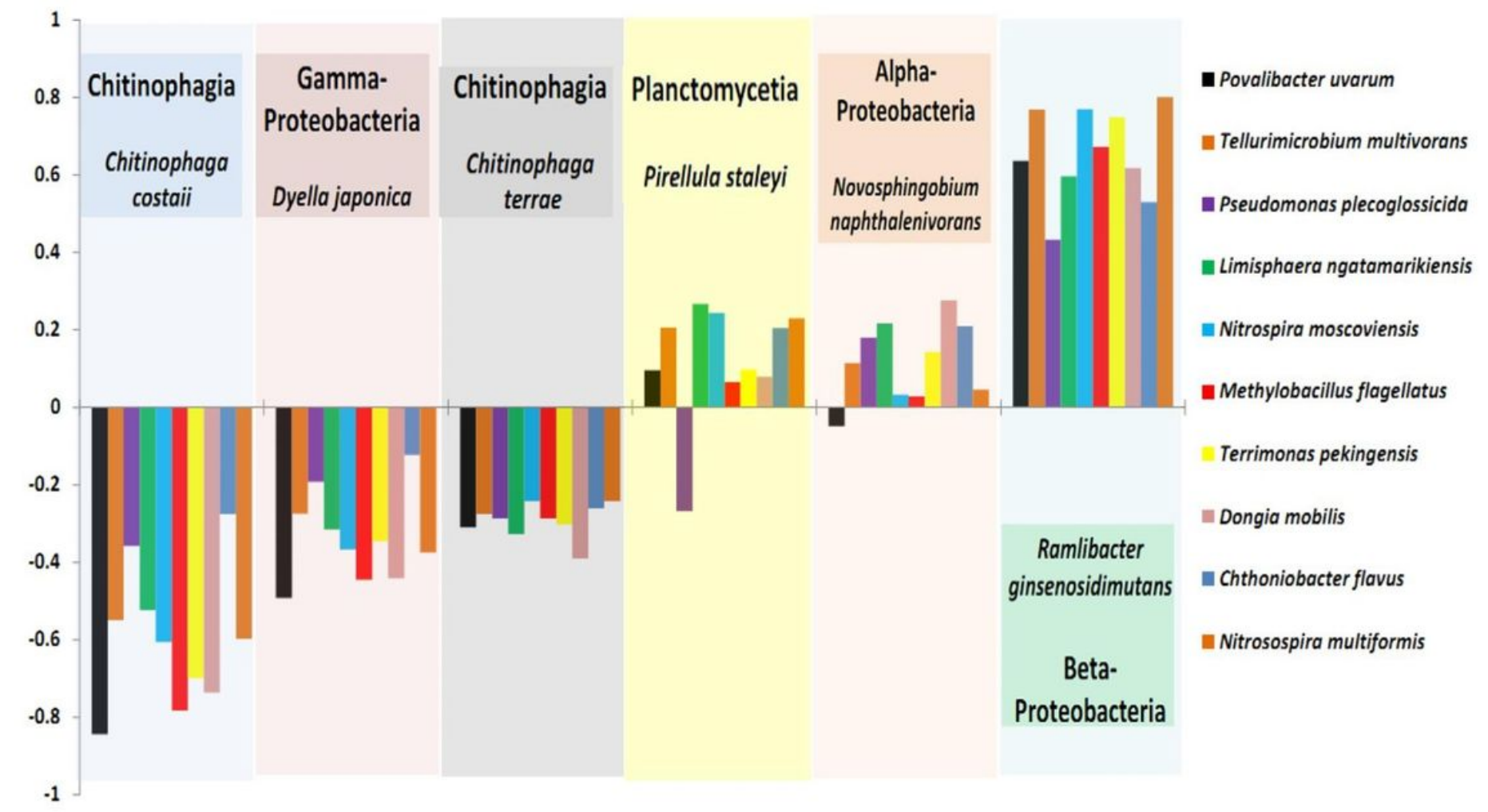

Figure 8

Histograms representing 10 positively and negatively co-occurred microbial Species in response to $\mathrm{C}$. costaii, C. terrae, D. japonica, P. staleyi, N.naphthalenivorans and R. nginsenosidimutans species found in the rhizo-compartments of both E. plantagineum (EP) and L. erythrorhizon (LE)

\section{Supplementary Files}

This is a list of supplementary files associated with this preprint. Click to download.

- SupplementaryFiguresS1S12.docx

- SupplementaryTablesS113.xIsx 